\title{
Concurrent Optimization of Consumer's Electrical Energy Bill and Producer's Power Generation Cost under a Dynamic Pricing Model
}

\author{
Tiansong Cui, Hadi Goudarzi, Safar Hatami, Shahin Nazarian, and Massoud Pedram \\ University of Southern California \\ Department of Electrical Engineering \\ Los Angeles CA USA \\ \{tcui, hgoudarz, shatami, shahin.nazarian,pedram\}@usc.edu
}

\begin{abstract}
Demand response is a key element of the smart grid technologies. This is a particularly interesting problem with the use of dynamic energy pricing schemes which incentivize electricity consumers to consume electricity more prudently in order to minimize their electric bill. On the other hand optimizing the number and production time of power generation facilities is a key challenge. In this paper, three models are presented for consumers, utility companies, and a third-part arbiter to optimize the cost to the parties individually and in combination. Our models have high quality and exhibit superior performance, by realistic consideration of non-cooperative energy buyers and sellers and getting real-time feedback from their interactions. Simulation results show that the energy consumption distribution becomes very stable during the day utilizing our models, while consumers and utility companies pay lower cost.
\end{abstract}

\section{INTRODUCTION}

There is no substitute for the status of electrical energy, which dramatically fuels both the development of economy and the improvement of people's living standard. Availability of affordable and sustainable electrical energy has been the key to prosperity and continued socioeconomic growth of nations and the world [1]. Two key characteristics of electrical energy are that it is easy to distribute but hard to store. More precisely, electrical energy can be transmitted to a faraway place with only a tiny loss, but unlike other common forms of energy such as chemical or kinetic, electricity must be used as it is being generated. If storage is needed, it must typically be converted immediately into another form of energy such as potential, kinetic, or electrochemical.

The huge difference between energy consumption levels at peak usage time and off-peak times has resulted in not only cost inefficiencies and potential brownouts and blackouts, but also environmental pollution due to over provisioning of the Power Grid and the resulting energy waste [7]. Utility companies are interested in reducing the peak demand of energy consumers so that their cost can be reduced. However, the power demand depends on exogenous factors and varies dramatically as a function of time of day and seasonal factors [10].

An ideal method to solve this problem is dynamic energy pricing [2]-[10]. Dynamic changes in energy prices provide an incentive for the customers to shift their energy consumption from peak-energy-use hours to off-peak hours, thus save money on their monthly electrical bill. At the same time, by proper use of energy, utility companies save capital expenditure by not having to add new power plants to the Grid in order to meet the customers' peak-hour demands. So, dynamic energy pricing can benefit both the consumer and the producer in an economical way.

Implementing dynamic energy pricing faces many challenges. The most difficult step is how to predict people's reaction to various dynamic energy pricing schemes, which calls for accurate models and practical algorithms. In addition, the price of electrical energy can have a significant effect on the national and local economies. Improper dynamic energy pricing can result in a decrease of economic activities or even economic dislocation. That is why governments impose restrictions on electrical energy prices.

Existing research on dynamic energy pricing can be classified into two categories: profit maximization for utility companies [10] or cost minimization for customers [7] and [8]. In reality, each of utility companies and customers tend to make their decisions based on the reaction of the other. Works such as the ones in [7], [8] and [10] fail to consider the feedback effects between the two, and cannot give a "closed loop" solution.

In the classical economics problems between sellers and buyers, economists always give suggestions to the sellers based on the reaction of the buyers or vice versa because although the government would like to maximize the total social welfare, we still need to consider sellers and buyers as non-cooperative and always making decisions based on their own best solution. This is also the case for energy users and utility companies. Considering this fact, three models of dynamic energy pricing are presented in this paper to solve the cost minimization problem for either the 
energy consumers or the utility companies. In addition, another contribution of this paper is to present a third model which is a combination of the first two where a feedback system is created and managers can make their decisions based on the reaction of customers and power generations [11]-[12].

The remainder of this paper is organized as follows. In the next section, we present our models for optimizing the cost of both costumers and power generations. Section III reports the simulation results. The paper is concluded in Section IV.

\section{Models AND Cost OPtiMiZATION MethodS}

As stated above, three types of optimization problems are presented in this paper. For each problem, a related model is created and an optimal solution is discussed. A unified electricity bill is used in all the models.

The first model deals with task scheduling problems. Under the given daily price function, we act as a house owner to decide when to start each task in order to minimize the total electrical energy bill. The second model is for engineers in the utility company. The energy demand at each time is given and the problem is to decide whether to turn on or turn off power generation facilities to meet the energy requirements while minimizing the cost to the utility company. In the third model, we analyze the problem from a global manager's perspective to decide the price distribution in order to maximize the total social welfare. This time we assume that both customers and utility companies are making their own optimal choices and find a good solution based on repeatedly calling for the first two models.

\section{A. Model for Homeowners}

Figure 1shows an example of a task scheduling solution based on the given electricity price function. The height of the task box in this figure signifies the amount of power each household task consumes while running.

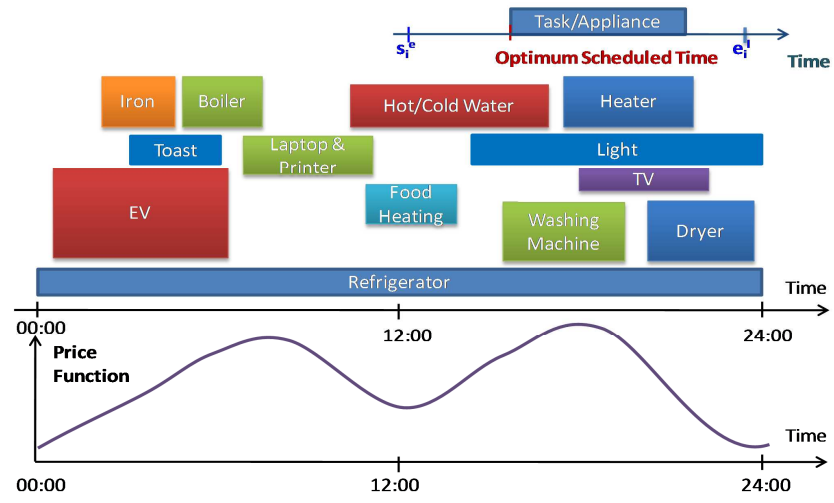

Figure 1. An example of the task scheduling problem.
In this paper, a slotted time model is assumed for all models, i.e., all system cost parameters and constraints as well as scheduling decisions are provided for discrete time intervals of constant length. The scheduling epoch is thus divided into a fixed number of equal-sized time slots (in the experiment, a day is divided into 24 time slots, each of duration 1 hour). Tasks can be launched only at the beginning of one of these time slots and will be completed at the end of the slots.

We define Price function, $P(i)$, as the price of one unit of energy $(\mathrm{kWh})$ at time slot $i$. In the first model, we assume that $P(i)$ is fixed and pre-announced by the utility company before the start of the day, which means house owners can make their decisions about the whole day but their decisions does not affect the energy price function.

In this model, we also assume that there are a number of tasks in each house that should be executed daily. These tasks are identified by index $j$. The set of task indexes is denoted by $K=\{1, \ldots, N\}$. For each task $j$, the earliest start time, es $(j)$, the latest end time, $l e(j)$, energy consumption per time slot, $C(j)$, and the duration of task, Time $(j)$, are specified.

To solve the task assigning problem, two additional definitions are needed: start time, $S(j)$, which represents the time slot when a task starts and task operation matrix, $M(i, j)$,which represents the operating condition of each task $\mathrm{j}$ at time slot $\mathrm{i}$. We set $M(i, j)=1$ when at time slot $i$, task $j$ is operating. Otherwise $M(i, j)=0$.

Using the above definition, the homeowner's cost minimization problem can be modeled as follows. Given $P(i), C(j)$, and Time $(j), e s(j), l e(j)$, we are to assign $S(j)$ for each $j$. The problem is to minimize the total cost

subject to:

$$
\text { Cost }_{h}=\sum_{i} \sum_{j} P(i) C(j) M(i, j)
$$

$$
\begin{aligned}
& S(j) \geq e s(j) \\
& S(j)+\operatorname{Time}(j)<l e(j)
\end{aligned}
$$

where $M(i, j)$ can be found by the following method:

Initialize $M(i, j)=0$ for all $i$ and $j$;

for each $j\{$

$$
\begin{aligned}
& \text { for }(i=S(j), i<S(j)+T(j), i++) \\
& M(i, j)=1
\end{aligned}
$$$$
\text { \} }
$$

In this model, for simplicity, we assume that each task is independent of other tasks. We use a greedy algorithm to find the minimal cost: for Time $(j)=k$, from the earliest start time to the latest possible start time, we calculate all the values of $P(i)+P(i+1)+\ldots+P(i+k-1)$ and find the minimal sum. Then we put this task into these timeslots. Repeat the above steps until all the tasks are arranged. It can simply be proven that the proposed greedy algorithm obtains the global optimum solution. 


\section{B. Model for Utility Companies}

As stated earlier, utility companies have been willing to reduce the peak demand from energy consumers. But when the demand is given, the engineers in the utility company should decide whether to turn on or turn off the power generation facilities. Many times a utility company prefers to waste some amount of energy in order to avoid repeatedly turning its power generation on and off, and thus, avoid the resulting large amount of startup operating costs. For example, in Los Angeles, some buildings in the downtown area may turn on their lights all night and waste energy although there is no body inside. This will be done to reduce the power generation startup cost.

In this model, we act as an engineer to consider how many power generation facilities will operate at each time, $T(i)$, when the energy demand for each time, Con(i), is given. For a utility company, we assume that the operating price of one power generation facility per time, $P_{c}$, the price of turning on one power generation facility, $P_{\text {on }}$, the price of turning off one power generation facility, $P_{\text {off }}$, and the amount of energy one power generation facility can offer, i.e., the load it can service are specified.

To solve this problem, we assign each power generation facility an integer number from 1 to Max-number. We can then create a power generation operation matrix $L(i, j)$, which represents the operating condition of each power generation $\mathrm{j}$ at time slot $\mathrm{i}$. We set $L(i, j)=1$ if power generation facility $j$ is operating at time slot $i$, Otherwise $L(i, j)=0$. For simplicity, we assume that we can turn on a power generation facility with the smallest number and turn off an operating power generation facility with the largest number. This means we will never have $L(i, j)=0$ but $L(i, j+1)=1 . L(i, j)$ and $T(i)$ can be translated from each other.

Our goal is to minimize the total cost of the utility company (namely $\operatorname{cost}_{g}$ ), which consists of the total operating cost and the total turning on and off cost. The problem formulation is as follows:

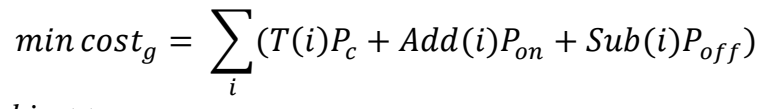

subject to:

$$
\begin{gathered}
\operatorname{Add}(i) \geq 0 \\
\operatorname{Add}(i) \leq T(i)-T(i-1) \\
\operatorname{Sub}(i) \geq 0 \\
\operatorname{Sub}(i) \leq T(i-1)-T(i) \\
T(i) \text { load } \geq \operatorname{Con}(i) \forall i
\end{gathered}
$$

where the last constraint forces the amount of energy provided by the power generations in each time to be greater than amount of energy needed at that time.

For this model, we use an algorithm that we refer to as the filling method. This method can be proven to find the optimal solution. The steps of this algorithm are following.
1. Calculate the minimal $T(i)$ based on the constraints and calculate the corresponding $L(i, j)$ :

For each $i$

$$
\begin{aligned}
& T[i]=\operatorname{Con}[i] / \text { load }+1 ; \\
& \text { for }(j=1 ; j<T[i]+1 ; j++) \\
& L[i][j]=1 ; \\
& \text { for }(j=T[i]+1 ; j<\text { number of time slots; } j++) \\
& L[i][j]=0 ; \\
& \text { \} }
\end{aligned}
$$

2. Calculate the number of time slots needed to be filled: expense $=\left(P_{\text {on }}+P_{\text {off }}\right) / P c$

3. Fill $L(i, j)$ by change several 0 to 1 :

$$
\begin{aligned}
& \text { for }(k=0 ; k<\text { expense } ;++)\{ \\
& \text { for each } i, j\{ \\
& \text { if }(L[i][j]==1) \\
& \begin{array}{l}
\{\quad \\
\quad
\end{array} \\
& \quad \text { if }(L[i+2+k][j]==1) \\
& \quad \text { for }(1=0 ; 1<\mathrm{k}+1 ; 1++) \\
& \quad L[\mathrm{i}+1+1][j]=1 ; \\
& \quad\} \\
& \quad\}
\end{aligned}
$$

4. Translate $L(i, j)$ to $T(i): T(i)=\sum_{j} L(i, j)$

\section{Model for Global Controller}

A solution for each of the above problems is proposed to reach a minimized cost for homeowners or power generations. But these models are far from complete because our goal is to maximize the social welfare. Also note that there exists a connection between homeowners and utility companies so that one's action exerts an impact to the other. Considering this, a feedback system is required.

Homeowners and utility companies are generally noncooperative and always make their own optimal decision. Based on this, what we should be acting like a global controller that manages the whole system where everyone inside the system is making its own choice.

Unlike our first model, this model does not assume a fixed price function, $P(i)$. Instead, the price is what the third-part arbiter should decide, although still preannounced to homeowners. However, since energy price is very sensitive to the national and local economies, almost every national or local government will impose price constraints on utility companies. In this model, we consider two price constraints explained below.

First, Average price $P$ is a price that government gives in order to regulate the profit of a utility company. For the company to arrange the price as a function of time slot, it should have: 


$$
\frac{1}{i} \sum_{i} P(i) \leq P_{\text {ave }}
$$

In addition, $P_{\max }$ and $P_{\min }$ are given to regulate the upper and the lower bounds of price at each time.

$$
P_{\min } \leq P(i) \leq P_{\max }
$$

Differently from the second model in this paper, the energy consumption of each time Con $(i)$ is not fixed. Instead, we should calculate this value after all the homeowners have made their decisions i.e.,

$$
\operatorname{Con}(i)=F i x(i)+\sum_{j} C(j) M(i, j)
$$

where Fix(i) represents the fixed energy consumption at each time.

The objective function will then be to maximize the social welfare, which means minimizing the total cost. Considering that the cost for homeowners will be the income of power generations, we take the cost function of the second model as our final cost, except for an additional consideration: the maximal number of power generation facilities, $T_{\max }$ ), is needed. For a relatively long time consideration, saving one power generation means saving space, human and repair cost. As a result, we take the cost function as:

$$
\text { Cost }=\operatorname{Cost}_{g}+\text { aTmax }
$$

where $a$ is a factor that represents the total fixed cost for having one power generation. So the problem of maximizing the social welfare is as follows:

Minimize

Cost $=a T_{\max }+\sum_{i}\left(T(i) P_{c}+\operatorname{Add}(i) P_{o n}+\operatorname{Sub}(i) P_{\text {off }}\right)$ subject to:

$$
\begin{gathered}
A d d(i) \geq 0 \\
\text { Add }(i) \leq T(i)-T(i-1) \\
\operatorname{Sub}(i) \geq 0 \\
\operatorname{Sub}(i) \leq T(i-1)-T(i) \\
T(i) \text { load } \geq F i x(i)+\sum_{j} C(j) M(i, j) \forall i \\
T(i) \leq T_{\max } \\
\frac{1}{i} \sum_{i} P(i) \leq P_{\text {ave }} \\
P(i) \leq P_{\text {max }} \\
P(i) \geq P_{\text {min }}
\end{gathered}
$$

In this problem, $\mathrm{P}(\mathrm{i})$ changes the behavior of the customers and affects the electricity demand in each time, and $T_{\max }$.

The problem of minimizing the Cost is an NP complete problem and we use simulated annealing to find a nearlyoptimal solution. Details of this method are as follows.

1. Set all $P(\mathrm{i})=P \_$ave
2. Based on given $P(i)$, call the homeowner model, assign all tasks and calculate total energy consumption of each time $\operatorname{Con}(i)$

3. Based on the calculated Con $(i)$, all the engineer model, assign each power generation, calculate the total cost

4. Randomly change the price distribution $P(i)$ within the constraints, repeat step 2 and 3 and calculate cost_new

5. If cost_new<cost, accept the new solution, if not accept in a certain probability based on the temperature $T$

6. Cool down and repeat from step 2 until $T$ reaches a certain value.

\section{Simulation Results}

To demonstrate the effectiveness of the proposed algorithms, cases corresponding to the aforesaid pricing models are examined.

In these simulations, duration of a time slot is set to one hour. For this reason, the minimum duration of a task is also set to one hour, and the durations of tasks are integer multiples of one hour. Moreover, power consumption of the tasks is determined with a granularity of one hour.

The proposed algorithms have been implemented in $\mathrm{C}++$ code and tested for random cases.

In Table I, we act as a house owner and we assume that there are in total 10 tasks for us to assign. By using the algorithm provided above, the cost has been reduced by about $13.3 \%$ in average. Figure 1 shows the initial solution and the final solution.

Table I. Cost Minimization for House Owners

\begin{tabular}{|c|c|c|c|}
\hline & Initial cost & Final cost & $\begin{array}{c}\text { Cost } \\
\text { reduction }\end{array}$ \\
\hline Expr. 1 & 1838 & 1594 & $13.3 \%$ \\
\hline
\end{tabular}
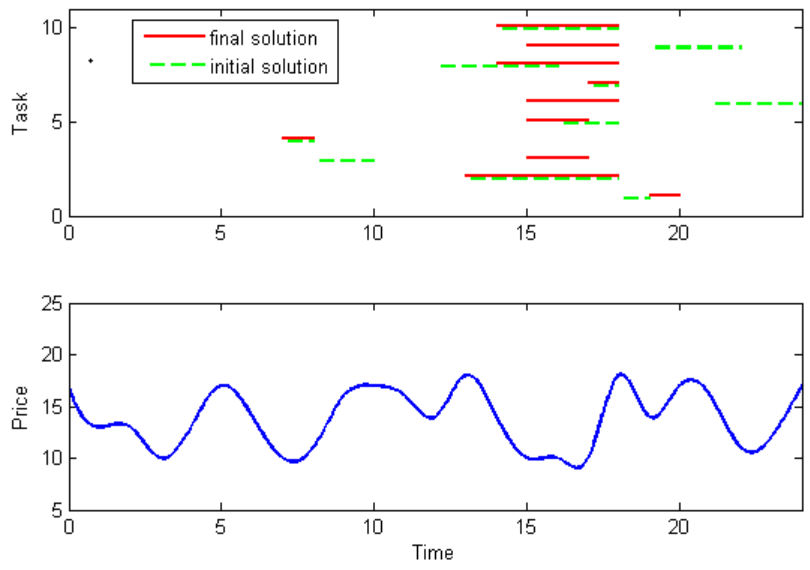

Figure 2.Task assignment process 
Figure 2 shows the task assignment process. Initially, all the tasks are assigned randomly. But after using our task assigning method, all the tasks are assigned at the lowestcost time slots based on meeting the earliest start time and latest end time constraints. Remember that the proposed greedy algorithm is optimal and its run time on a normal machine for more than 100 tasks is less than a second.

In Table II, we tested the model under three different values for expense. The results show that as the value of expense increases, the cost minimization effectiveness is enhanced from $5.2 \%$ to $26.3 \%$.

Table II. Cost Minimization for Utility Companies

\begin{tabular}{|c|c|c|c|}
\hline & Initial cost & Final cost & $\begin{array}{c}\text { Cost } \\
\text { reduction }\end{array}$ \\
\hline expense $=1$ & 2223 & 2107 & $5.2 \%$ \\
\hline expense $=2$ & 2748 & 2303 & $16.2 \%$ \\
\hline expense $=3$ & 3713 & 2738 & $26.3 \%$ \\
\hline
\end{tabular}

Figure 3 shows how our algorithm helps to assign the fill-up power generations for different value of expense.

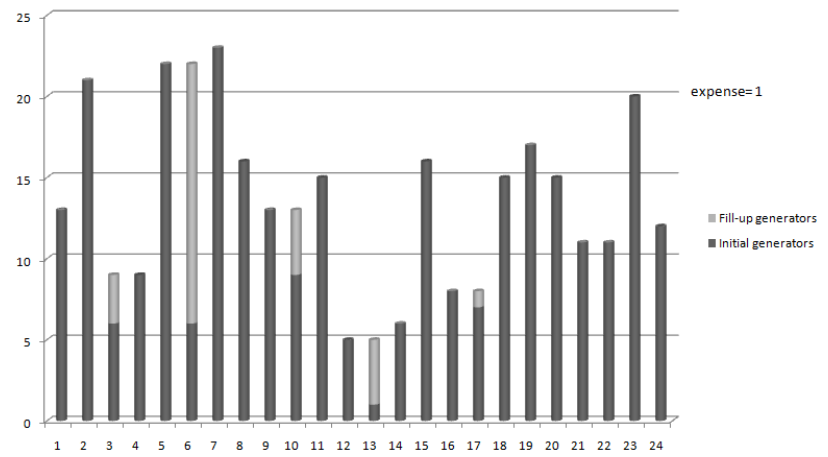

(a)

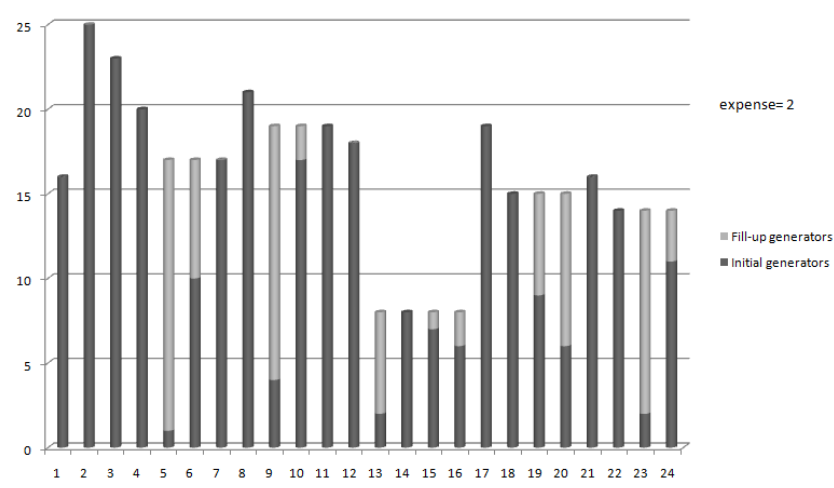

(b)

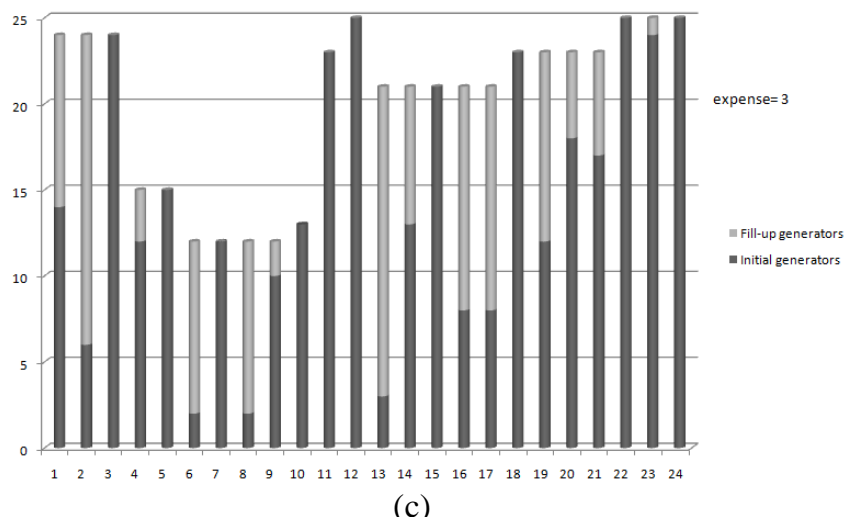

(c)

Figure 3.Power generation working conditions for different expense profiles

In Table III, we use simulated annealing for cost minimization. We assume that 1 power generation serves 10 houses and each house has 10 tasks so that there are 100 tasks in total. We can see from the tables that both homeowners and power generations have significantly reduced their cost.

Table III. Cost Minimization for Combined Model

\begin{tabular}{|c|c|c|c|}
\hline & Base-line cost & Final cost & $\begin{array}{c}\text { Cost } \\
\text { reduction }\end{array}$ \\
\hline $\begin{array}{c}\text { Utility } \\
\text { companies }\end{array}$ & 6939 & 4256 & $38.7 \%$ \\
\hline Homeowners & 350200 & 293750 & $16.1 \%$ \\
\hline
\end{tabular}

In this table, base-line cost refers to the cost in a case with initial scheduling for tasks in the homeowners and initial state for power generations.

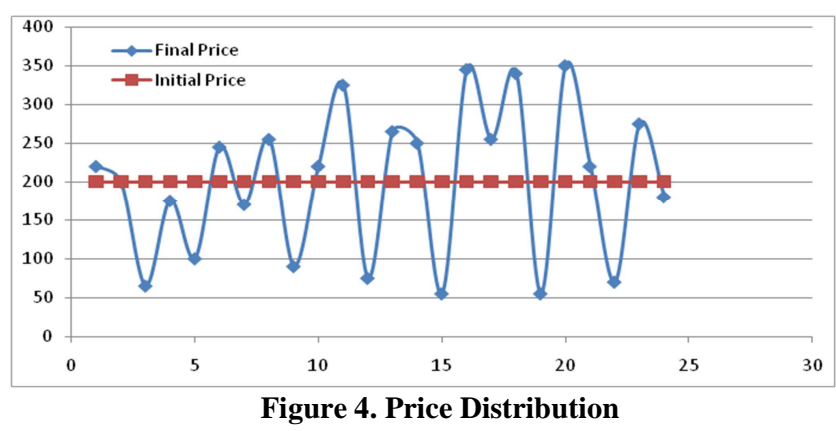

Figure 4 shows the change of price distribution. Initially, we set average price to all the time slots. After simulated annealing, the price distribution varies up to $\pm 75 \%$. 


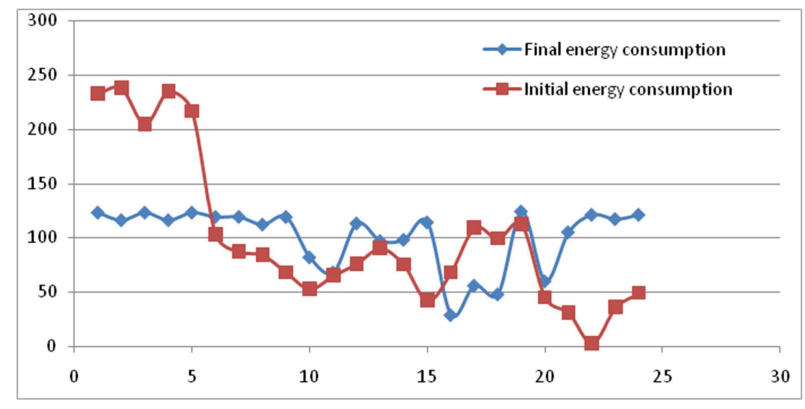

Figure 5.Energy consumption distribution

Figure 5 shows the change of energy consumption distribution. Initially, the energy consumption exhibits peak and off-peak time behavior. But finally, the energy consumption distribution turns out to be relatively flat during the day. The energy consumption at the peak time is reduced about $50 \%$.

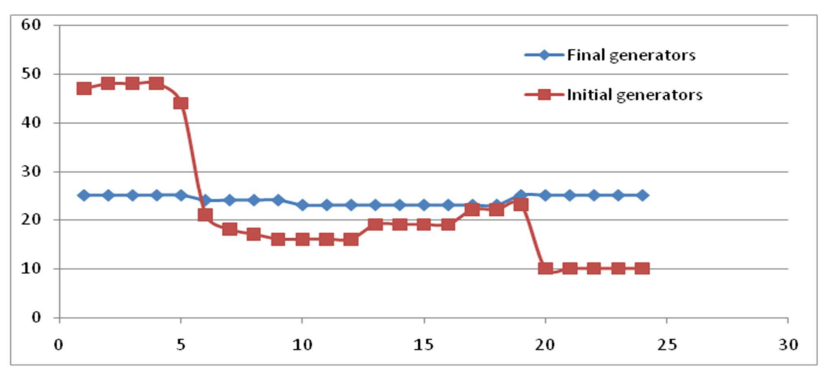

Figure 6. Power generation distribution

Figure 6 shows the change of power generation distribution. Initially, we have to turn on a lot of power generation facilities in the peak time and turn off at the offpeak time. But finally, as the energy consumption turns out to be relatively flat, the number of power generation facilities needed to be turned on or turned off becomes small. The maximum number of power generation facilities is reduced by about $50 \%$.

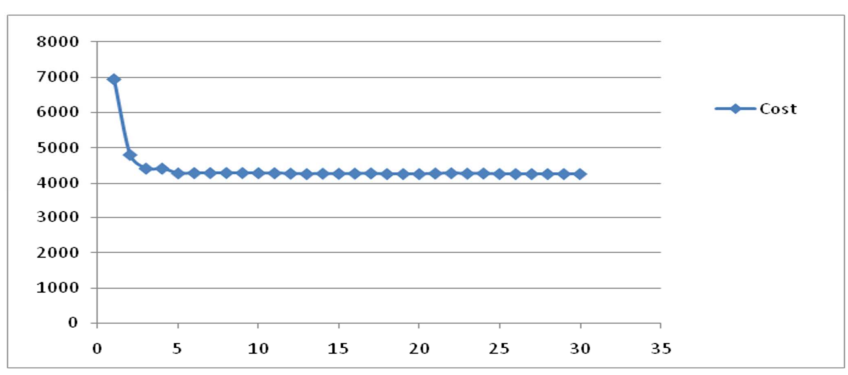

Figure 7. Simulated Annealing Steps

Figure 7 shows the change of total cost as a function of the simulated annealing steps. The cost reduces significantly in the first steps and comes relatively stable in the rest. The cost reduces down to about $40 \%$ of the baseline solution.
Runtime of the proposed heuristic for the third model is less than 10 seconds for 100 aggregated task in house owners for a machine with a dual core processor with frequency of $2.80 \mathrm{GHz}$. This run time is acceptable for using this algorithm real-time.

\section{CONCLUSION}

Three different models of cost minimization including their problem formulation and solution were presented. All three models were implemented and tested for some random test schemes. More specifically in our key model, customers and power generation facilities are simultaneously considered as non-cooperative, always making decisions based on their own best solutions. A feedback system is utilized such that a manager can make the best decision according to the action and reaction of utility companies and customers. The results were compared to a base-line solution with significant improvements.

\section{REFERENCES}

[1] L. D. Kannberg, D. P. Chassin, J. G. DeSteese, S. G. Hauser, M. C. Kintner-Meyer, R. G. Pratt, L. A. Schienbein, and W. M. Warwick, "GridWise ${ }^{\mathrm{TM}}$ : The Benefits of a Transformed Energy System," PNNL-14396, Pacific Northwest National Laboratory, Richland, Sep. 2003.

[2] S. Caron and G. Kesidis, "Incentive-based Energy Consumption Scheduling Algorithms for the Smart Grid," Proc. Smart Grid Communications Conf., 2010.

[3] P. Samadi, H. Mohsenian-Rad, R. Schober, V. Wong, and J. Jatskevich, "Optimal real-time pricing algorithm based on utility maximization for smart grid," Proc. Smart Grid Communications Conf., 2010.

[4] L. Chen, S. Low, and J. Doyle, "Two market models for demand response in power networks," Proc. Smart Grid Communications Conf., 2010.

[5] D. O'Neill, M. Levorato, A. Goldsmith, and U. Mitra, "Residential demand response using reinforcement learning," Proc. Smart Grid Communications Conf., 2010.

[6] S. Kishore, L. V. Snyder, "Control Mechanisms for Residential Electricity Demand in Smart Grids," Proc. Smart Grid Communications Conf., 2010.

[7] S. Hatami and M. Pedram, "Minimizing the Electricity Bill of Cooperative Users under a Quasi-Dynamic energy pricing Model," Proc. Smart Grid Communications Conf., 2010.

[8] H. Goudarzi, S. Hatami, and M. Pedram, "Demand-side load scheduling incentivized by dynamic energy prices," Proc. 2nd Int'l Conf. on Smart Grid Communications, Oct. 2011.

[9] http://www.powersmartpricing.org/tools

[10] Tariq Samad, Technology Developments and R\&D Challenges for Smart Grid Applications in Homes, Buildings, and Industry, Presentation slides.

[11] N. Gregory Mankiw, Principle of Economics, Dryden Pr, 1997.

[12] Tikuan Gu, Thermal Power Station, China Power Press, 2008. 